\title{
INCLUSÃO DE FENOS DE FOLHA DE LEUCENA E DE CUNHÃ NA RAÇÃO DE POEDEIRAS
}

\author{
INCLUSION OF LEUCAENA LEUCOCEPHALA AND CLITORIA TERNATEA LEAF HAYS \\ INTO LAYING HEN DIET
}

\author{
Lopes, I.R.V. ${ }^{1 *}$; Freitas, E.R. ${ }^{2}$; Nascimento, G.A.J.2; Viana Neto, J.L. ${ }^{3}$; Cruz, C.E.B. ${ }^{2}$ \\ e Braz, N.M. ${ }^{2}$
}

\begin{abstract}
${ }^{1}$ Universidade Federal do Ceará. Curso de Agronomia. Campus Cariri. Crato, CE. Brasil. *iranirvl@cariri.ufc.br ${ }^{2}$ Universidade Federal do Ceará. Departamento de Zootecnia. Fortaleza, CE. Brasil.

${ }^{3}$ Instituto Federal de Educação, Ciência e Tecnologia do Estado do Ceará. Tauá, CE. Brasil.
\end{abstract}

\section{PaLAVRAS ChAVE ADICIONAIS}

Desempenho. Leguminosas. Qualidade dos ovos.

\section{RESUMO}

Objetivou-se comparar o efeito da inclusão de fenos de folha de leucena (FFL) e de cunhã (FC) na ração, sobre o desempenho e a qualidade dos ovos de poedeiras. Cem poedeiras Hisex Brown (64 semanas de idade) foram distribuídas em um delineamento inteiramente casualizado, com 5 tratamentos e 5 repetições de 4 aves. Por 63 dias (3 períodos de 21 dias), durante os quais as aves receberam ração e água à vontade, foram avaliados os fatores fonte de energia (milho ou sorgo) e o tipo de feno (leucena ou cunhã) e uma ração controle à base de milho e farelo de soja sem inclusão de feno em um fatorial $2 \times 2+1$. A postura, o peso dos ovos, a massa de ovo, a conversão alimentar, a proporção dos componentes do ovo e as unidades Haugh não sofreram influências dos tratamentos. As poedeiras alimentadas com ração controle, apresentaram o menor consumo. A inclusão de $F F L$, na ração contendo milho ou sorgo, resultou em maior pigmentação das gemas em relação à inclusão de FC. Independente do cereal utilizado como fonte de energia na formulação da ração de postura, a inclusão de FFL ou de FC resulta em desempenho semelhante, porém, o FFL apresenta maior capacidade de pigmentação das gemas.

\section{SUMMARY}

The effects of including leucena leaf (FFL) or cunhã (FC) hay into diet on performance and egg quality of 100 Hisex Brown (64 weeks old) laying

\section{AdDitional KEYWORDS}

Egg quality. Legume forages. Performance.

hens were compared. Birds were distributed into a completely randomized design with five treatments and five replicates of 4 birds each. The treatments were designed in a factorial $2 \times 2+1$, which evaluated the factors energy source (corn or sorghum) and type of hay (leucena or cunhã) and an a control diet based on corn and soybean meal without inclusion of hay. The trial lasted 63 days (three periods of 21 days), during which the birds received feed and water ad libitum. Treatments did significantly affect, neither production, weight, mass, component proportion and Haugh units of eggs, nor feed conversion. However, laying hens fed ration without hay (control diet) had the lowest consumption. The inclusion of $\mathrm{FFL}$, in the diet containing corn or sorghum, resulted in higher yolk pigmentation than inclusion of FC. The inclusion of FFL or FC, independent of the grain utilized as an energy source, results in a similar performance, but FFL increased yolk pigmentation capacity.

\section{INTRODUÇÃO}

O aumento dos preços do milho e do farelo de soja ou a baixa disponibilidade desses ingredientes tem incentivado pesquisas em busca de alimentos alternativos que reduzam os custos de produção das aves sem prejudicar o desempenho zootécnico. Porem a utilização de sorgo, milheto, 
quirera e farelo de arroz e farelo de coco, produtos mais baratos, na alimentação de poedeiras apresenta,entre outros problemas, a redução na pigmentação da gema dos ovos, conforme constatado por Braga et al. (2005) para o farelo de coco, Brum Júnior et al. (2007) para o farelo de arroz, Moreno et al. (2007) para o sorgo e Garcia et al. (2011) para o milheto. Dessa forma, quando são utilizados ingredientes deficientes de xantofilas, deve-se incluir pigmentos na ração para melhorar a coloração da gema do ovo, visto que a cor amarelada dos produtos avícolas é conseqüência direta da absorção dos carotenóides presentes na ração (Silva et al., 2000).

$\mathrm{Na}$ literatura são encontrados alguns relatos sobre o uso de fenos de plantas, como Gliricidia sepium (Odunsi et al., 2002), Lablab purpureus (Odunsi, 2003), Microdesmis puberula (Esonu et al., 2004) e Azadirachta indica (Esonu et al., 2005), na alimentação de poedeiras. Nessas pesquisas, os fenos foram incluídos como um ingrediente em rações formuladas para serem isonutrientes. Os resultados indicaram que sempre que aumentava a participação do feno na ração houve redução no desempenho das aves e melhora na coloração da gema dos ovos, entretanto, com a inclusão de 5 \% de feno na ração foi possível obter desempenho e qualidade dos ovos semelhantes aos resultados obtidos para as aves alimentadas sem a inclusão dos fenos na ração.

Entre as plantas adaptadas ao bioma caatinga, a leucena (Leucaena leucocephala) se destaca por ser uma leguminosa, cujo material foliar apresenta bom poder pigmentante (carotenóides), excelente aporte de proteína, satisfatório valor energético, porém possui limitação de inclusão dietética devido aos níveis de lignocelulose da fibra e uma substância fitotóxica denominada mimosina(Oliveira et al., 2000; Arruda et al., 2010). Outra cultura presente no semiárido nordestino com potencial forrageiro é a cunhã (Clitoria ternatea), leguminosa tropical perene, rústica, visto que é bastante resistente à seca (Alcântara e Bufarah, 1988). Por possuir caules finos e elevada massa foliar, é uma forrageira ideal para produção de feno apresentando bom nível protéico e pigmentante, representado por considerável teor de beta caroteno, que atua diretamente na coloração das gemas dos ovos e da carcaça de frangos de corte (Araújo Filho et al., 1981). Entretanto, o uso dessas plantas na alimentação de aves ainda é incipiente nessa região do Brasil.

Diante do exposto, a presente pesquisa teve como objetivo comparar o feno da folha de leucena (FFL) e o de cunhã (FC) como fonte de pigmentos na ração de poedeiras, através dos seus efeitos sobre o desempenho e a qualidade dos ovos.

\section{MATERIALE MÉTODOS}

Foram utilizadas 100 poedeiras comerciais da linhagem Hisex Brown, com 64 semanas de idade. As aves foram pesadas e alojadas aos pares em gaiolas de arame galvanizado $(25 \times 40 \times 30 \mathrm{~cm})$. Cada gaiola dispunha de bebedouro tipo nipple, comedouro tipo calha e coletor de ovos frontal.

As aves foram distribuídas em um delineamento inteiramente casualizado, com cinco tratamentos e cinco repetições de quatro aves. Os tratamentos foram concebidos segundo um fatorial $2 \times 2+1$, em que foram avaliados os fatores fonte de energia (milho ou sorgo) e tipo de feno (leucena ou cunhã) e um tratamento adicional, uma ração controle à base de milho e farelo de soja sem inclusão de feno.

Para a montagem das parcelas experimentais, antes do início do experimento, as aves foram selecionadas, com base no peso $(1,779 \pm 0,056 \mathrm{~kg})$ e na produção de ovos (94,05 $\pm 2,5 \%)$, e distribuídas uniformemente nas gaiolas, de modo que todas as repetições tivessem aves com pesos e produção de ovos similares, conforme recomendações de Sakomura e Rostagno (2007). 
As rações experimentais (tabela I) foram formuladas segundo as recomendações nutricionais constantes no manual de manejo da linhagem e a composição dos alimentos proposta por Rostagno et al. (2005), exceto, para o feno de leucena (Leucaena leucocephala).

Os valores médios de matéria seca (MS), proteína bruta $(\mathrm{PB})$, extrato etéreo (EE), fibra bruta (FB), fibra em detergente neutro (FDN), fibra em detergente ácido (FDA) e energia bruta (EB), expressos com base na matéria seca foram: 89,68 \% MS; 22,16\% PB; 5,70 \% EE; 12,31 \% FB; 57,59 \% FDN $24,05 \%$ FDA e $3600 \mathrm{kcal} / \mathrm{kg}$ EB para o feno da folha de leucena (FFL); e 91,90\% MS; 13,62 \% PB; 3,59\% EE; 31,41\% FB; 62,08\% FDN; 37,81 \% FDA e 4002 kcal/kg EB para o feno de cunhã (Clitoria ternatea) (FC). A inclusão dos fenos foi de 2 \% na ração, sendo considerados os valores de composição do FFL determinados e os sugeridos por D’Mello et al. (1987). Entretanto, pela falta de informações sobre o valor nutricional

Tabela I. Composição das dietas experimentais. (Composition of experimental diets).

\begin{tabular}{|c|c|c|c|c|c|}
\hline \multirow[b]{2}{*}{ Ingredientes } & \multirow[t]{2}{*}{ Controle } & \multicolumn{2}{|c|}{ Milho } & \multicolumn{2}{|c|}{ Sorgo } \\
\hline & & $2 \% \mathrm{FFL}$ & $2 \% \mathrm{FC}$ & $2 \% \mathrm{FFL}$ & $2 \% \mathrm{FC}$ \\
\hline Milho & 64,65 & 63,10 & 63,10 & 0,00 & 0,00 \\
\hline Sorgo (baixo tanino) & 0,00 & 0,00 & 0,00 & 60,96 & 60,96 \\
\hline Farelo de soja & 23,82 & 23,00 & 23,00 & 23,00 & 23,00 \\
\hline Calcário & 9,10 & 9,00 & 9,00 & 9,00 & 9,00 \\
\hline Fosfato mono-bicálcico & 1,56 & 1,57 & 1,57 & 1,56 & 1,56 \\
\hline Sal comum & 0,39 & 0,39 & 0,39 & 0,41 & 0,41 \\
\hline Suplemento vitamínico ${ }^{1}$ & 0,20 & 0,20 & 0,20 & 0,20 & 0,20 \\
\hline Suplemento mineral ${ }^{2}$ & 0,10 & 0,10 & 0,10 & 0,10 & 0,10 \\
\hline DL-metionina & 0,16 & 0,17 & 0,17 & 0,20 & 0,20 \\
\hline L-lisina & 0,02 & 0,02 & 0,02 & 0,05 & 0,05 \\
\hline Feno de folha de leucena (FFL) & 0,00 & 2,00 & 0,00 & 2,00 & 0,00 \\
\hline Feno de cunhã (FC) & 0,00 & 0,00 & 2,00 & 0,00 & 2,00 \\
\hline Óleo de soja & 0,00 & 0,45 & 0,45 & 2,52 & 2,52 \\
\hline Total & 100,00 & 100,00 & 100,00 & 100,00 & 100,00 \\
\hline \multicolumn{6}{|l|}{ Composição calculada } \\
\hline Energia metabolizável $\left(\mathrm{kcal} \mathrm{kg}^{-1}\right)$ & 2.700 & 2.700 & 2.700 & 2.700 & 2.700 \\
\hline Proteína bruta (\%) & 16,50 & 16,50 & 16,50 & 16,50 & 16,50 \\
\hline Lisina (\%) & 0,84 & 0,84 & 0,84 & 0,84 & 0,84 \\
\hline Metionina + cistina (\%) & 0,70 & 0,70 & 0,70 & 0,70 & 0,70 \\
\hline Metionina (\%) & 0,42 & 0,42 & 0,42 & 0,42 & 0,42 \\
\hline Treonina (\%) & 0,64 & 0,64 & 0,64 & 0,64 & 0,64 \\
\hline Triptofano total (\%) & 0,19 & 0,19 & 0,19 & 0,19 & 0,19 \\
\hline Cálcio (\%) & 3,90 & 3,90 & 3,90 & 3,90 & 3,90 \\
\hline Fósforo disponível (\%) & 0,40 & 0,40 & 0,40 & 0,40 & 0,40 \\
\hline Sódio (\%) & 0,19 & 0,19 & 0,19 & 0,19 & 0,19 \\
\hline
\end{tabular}

${ }^{1}$ Composição por kg do produto: Vit. A: 3500000 Ul; Vit. B1, 1000 mg; Vit. B2: 1500 mg; Vit. B12: 4000 mg; Vit. D3: 750000 Ul; Vit. E: 2000 mg; Vit. K3: 1000 mg; Cloreto de colina: 250 mg; Niacina: 7500 mg; Selênio: $150 \mathrm{mg}$; Pantotenato de cálcio: $2500 \mathrm{mg}$; Antioxidante: $25 \mathrm{~g}$; Veículo: q.s.p. $1000 \mathrm{~g}$.

${ }^{2}$ Composição por kg do produto: Mn: 65 g; Zn: 50 g; Fe: 50 g; Cu: 12 g; I: 1 g; Veículo: q.s.p. 1000 g. 
do FC para aves, optou-se pela substituição isométrica do FFL pelo FC.

O ensaio teve a duração de 63 dias, sendo dividido em 3 períodos de 21 dias, durante os quais as aves receberam ração e água à vontade. Os comedouros foram abastecidos duas vezes ao dia (às $08 \mathrm{~h} 00 \mathrm{~min}$ e às $16 \mathrm{~h} 00 \mathrm{~min}$ ), tendo-se o cuidado de evitar desperdício de ração. O programa diário de luz utilizado foi de 16 horas (natural e artificial) e a coleta de ovos foi feita diariamente às $15 \mathrm{~h} 00 \mathrm{~min}$.

Durante todo o período experimental, a temperatura e a umidade relativa do ar dentro do galpão foram registradas diariamente às 08 e às $16 \mathrm{~h}$ através de termômetro de máxima e mínima e de termohigrômetro.

As variáveis de desempenho estudadas foram: consumo de ração (g/ave/dia), porcentagem de postura (\%), peso do ovo (g), massa de ovo (g/ave/dia) e conversão alimentar.

A quantidade de ração consumida em cada período de 21 dias foi determinada através da pesagem da ração fornecida e das sobras. O controle da produção foi realizado diariamente, por meio de registro do número de ovos coletados por gaiola e, ao final de cada período, dividindo-se o total de ovos produzidos pelo número médio de aves de cada parcela, foi calculada a percentagem de postura.

Uma vez por semana, todos os ovos coletados foram armazenados à temperatura de $18{ }^{\circ} \mathrm{C}$, sendo pesados na manhã seguinte em balança eletrônica de precisão 0,01 g, para determinação do peso médio do ovo. A partir desse dado, calculou-se a massa de ovos. A conversão alimentar foi expressa como ração consumida/massa de ovo produzida.

À medida que cada ovo era quebrado sobre uma superfície plana de vidro, foram realizadas a determinação da coloração da gema, através do leque colorimétrico (DMS), e a medição da altura do albúmen, com o auxílio de um micrômetro digital. Em seguida, as gemas eram separadas do albúmen e pesadas.

As cascas foram lavadas em água corrente e postas para secar a sombra, em temperatura ambiente, por 48 horas, e, posteriormente, pesadas.

Os valores de unidade Haugh foram obtidos por meio da relação logarítmica entre o peso do ovo (W) e a altura do albúmen $(\mathrm{H})$, com a aplicação dos dados à fórmula:

$$
\mathrm{UH}=100-\log \left(\mathrm{H}+7,57-1,7 \mathrm{~W}^{0,37}\right)
$$

As percentagens de gema ( $G$ ) e de casca (C) foram calculadas dividindo-se o peso médio de cada constituinte pelo peso médio do ovo e multiplicando-se o valor obtido por 100. A percentagem de albúmen (A) foi obtida por diferença [100 - (\%G + \%C)].

Os dados foram submetidos à análise de variância para avaliar o efeito dos tratamentos e testar o fatorial por meio do Statistical Analysis System (SAS Institute, 2000). O teste de Dunnett a $5 \%$ de probabilidade foi utilizado para se comparar o tratamento controle em relação a cada um dos demais tratamentos e o teste $t$ a $5 \%$ de probabilidade foi utilizado para se comparar os fatores avaliados, fonte de energia e tipo feno.

\section{RESULTADOSEDISCUSSÃO}

A temperatura média durante os experimentos foi de $29,38{ }^{\circ} \mathrm{C}$, $\left(32,73 \pm 0,66{ }^{\circ} \mathrm{C}\right.$ máxima e $26,04 \pm 0,64^{\circ} \mathrm{C}$ mínima). A média da umidade relativa foi de $65 \pm 0,64 \%$.

Os resultados de desempenho das poedeiras são apresentados na tabela II. A percentagem de postura, o peso dos ovos, a massa de ovo e a conversão alimentar não sofreram influências dos tratamentos. Entretanto, o consumo de ração variou significativamente entre os tratamentos.

Quando se avaliou o efeito da fonte de energia (milho ou sorgo) e do tipo de feno (FFL ou FC), observou-se que não houve interação significativa desses fatores para as variáveis de desempenho. Também se 
verificou que, a fonte de energia ou o tipo de feno na ração não influenciaram os resultados obtidos.

As aves alimentadas com ração sem feno (controle) apresentaram o menor consumo em relação aos demais tratamentos.

O aumento no consumo com a inclusão de feno nas rações pode ser associado à tentativa das poedeiras em regular a ingestão de energia. Segundo Janssen and Carré (1989), o incremento do teor de fibra na ração pode promover redução da digestibilidade de nutrientes e, consequentemente, reduzir a energia metabolizável da ração, o que resulta em aumento no consumo.

O aumento do nível de fibra, resultante da inclusão de fenos de folhas de diferentes espécies vegetais típicas de regiões tropicais, em rações tradicionais formuladas com milho para poedeiras pode influenciar o consumo de ração das aves, aumentando ou diminuindo a ingestão (Odunsi et al., 2002; Odunsi, 2003; Esonu et al., 2005).

A ingestão de energia controla a produção de ovos, independente do consumo de proteína ou de aminoácidos. Foram observadas alterações no peso dos ovos produzidos quando ocorrem mudanças nos níveis protéicos ou de alguns aminoácidos específicos como metionina e lisina, e nos teores de ácido linoléico da dieta de poedeiras (Leeson and Summers, 2001).

Nesse contexto, pode-se inferir que, os ajustes no consumo apresentados pelas aves alimentadas com as rações contendo

Tabela II. Desempenho de poedeiras alimentadas com feno da folha de leucena (FFL) e de cunhã (FC). (Performance of laying hens fed diets containing leucena leaf (FFL) and cunhã (FC) hay).

\begin{tabular}{|c|c|c|c|c|c|}
\hline & $\begin{array}{l}\text { Consumo } \\
\text { g/ave/dia }\end{array}$ & $\begin{array}{c}\text { Postura } \\
\%\end{array}$ & $\begin{array}{c}\text { Peso ovo } \\
\mathrm{g}\end{array}$ & $\begin{array}{c}\text { Massa de ovo } \\
\text { g/ave/dia }\end{array}$ & $\begin{array}{c}\text { Conversão } \\
\text { alimentar }\end{array}$ \\
\hline \multicolumn{6}{|l|}{ Tratamentos } \\
\hline Controle & 102,36 & 91,34 & 63,93 & 58,61 & 1,75 \\
\hline Milho + $2 \%$ FFL & $106,73^{*}$ & 92,18 & 65,48 & 58,74 & 1,82 \\
\hline Milho $+2 \%$ FC & $107,35^{\star}$ & 92,16 & 65,14 & 59,86 & 1,80 \\
\hline Sorgo + $2 \%$ FFL & $105,86^{*}$ & 90,51 & 63,67 & 59,88 & 1,77 \\
\hline Sorgo $+2 \% \mathrm{FC}$ & $106,32^{*}$ & 94,03 & 63,45 & 63,21 & 1,69 \\
\hline Média & 105,72 & 92,04 & 64,73 & 60,06 & 1,76 \\
\hline CV (\%) & 2,16 & 4,05 & 4,43 & 5,02 & 5,44 \\
\hline \multicolumn{6}{|l|}{ Fonte de energia } \\
\hline Milho & 107,04 & 92,27 & 65,31 & 59,30 & 1,81 \\
\hline Sorgo & 106,09 & 92,17 & 64,56 & 61,55 & 1,73 \\
\hline \multicolumn{6}{|l|}{ Tipo de feno } \\
\hline FFL & 106,29 & 91,34 & 64,57 & 59,30 & 1,79 \\
\hline $\mathrm{FC}$ & 106,83 & 93,10 & 65,29 & 61,54 & 1,74 \\
\hline \multicolumn{6}{|l|}{ Efeitos - ANOVA p-valor } \\
\hline Tratamento & 0,0214 & 0,6566 & 0,7664 & 0,1483 & 0,2978 \\
\hline Fonte de energia (FE) & 0,4028 & 0,9556 & 0,5956 & 0,1408 & 0,1200 \\
\hline Tipo de feno (TF) & 0,6318 & 0,3266 & 0,6094 & 0,1445 & 0,2927 \\
\hline FE $\times$ TF & 0,9432 & 0,3214 & 0,4531 & 0,4559 & 0,5449 \\
\hline
\end{tabular}

$C V=$ Coeficiente de variação. *Diferente em relação ao controle pelo teste de Dunnet $(p<0,05)$. 
fenos foram suficientes para garantir o suprimento dos nutrientes necessários à manutenção da produção e do peso dos ovos. Por consequência, a massa de ovo, também não foi afetada pelos tratamentos.

Estudos desenvolvidos para avaliar a influência de fenos de folhas de diferentes plantas no desempenho de poedeiras têm apresentado resultados variáveis. Odunsi (2003) observou decréscimo no consumo e na produção de ovos de poedeiras que receberam 10 e $15 \%$ de inclusão de feno de Lablab purpureus na ração, sem alteração na conversão alimentar. Odunsi et al. (2002) verificaram diminuição na ingestão de ração e na produção de ovos à medida que os níveis de feno da folha de Gliricidia sepium se elevaram, o que resultou em piora na conversão alimentar. Por outro lado, Esonu et al. (2005) notaram que o consumo e a produção de ovos cresceram gradativamente com o incremento dos níveis de inclusão de folhas de neem (Azadirachta indica), porém, o peso dos ovos e a conversão alimentar não foram afetadas.

Quando se avaliou (tabela III) o efeito da fonte de energia (milho ou sorgo) e do tipo de feno (FFL ou FC), observou-se que não houve interação significativa desses fatores para os constituintes e a qualidade dos ovos. Também se constatou que a fonte de energia ou o tipo de feno na ração não influenciaram na proporção dos componentes do ovo e nem nos valores de Unidade Haugh, entretanto afetaram a pigmentação das gemas dos ovos.

Conforme Grobas y Mateos (1996) a proporção relativa de gema, casca e albúmen

Tabela III. Qualidade dos ovos de poedeiras alimentadas com feno de leucena (FFL) e de cunhã (FC). (Egg quality of hens fed diets containing leucena (FFL) or cunhã (FC) hay).

\begin{tabular}{|c|c|c|c|c|c|}
\hline & Gema (\%) & Albúmen (\%) & Casca (\%) & Unidades Haugh & Cor da gema \\
\hline \multicolumn{6}{|l|}{ Tratamentos } \\
\hline Controle & 23,93 & 66,65 & 9,42 & 76,54 & 7,95 \\
\hline Milho + $2 \%$ FFL & 24,22 & 66,46 & 9,32 & 77,64 & $9,74^{*}$ \\
\hline Milho $+2 \%$ FC & 24,29 & 66,62 & 9,09 & 77,99 & $7,60^{*}$ \\
\hline Sorgo + $2 \%$ FFL & 25,03 & 65,81 & 9,16 & 76,18 & $5,76^{*}$ \\
\hline Sorgo + $2 \% \mathrm{FC}$ & 23,90 & 67,04 & 9,06 & 77,73 & $3,21 *$ \\
\hline Média & 24,27 & 66,52 & 9,21 & 76,62 & 6,85 \\
\hline CV (\%) & 5,23 & 2,07 & 2,97 & 5,77 & 2,40 \\
\hline \multicolumn{6}{|l|}{ Fonte de energia } \\
\hline Milho & 24,25 & 66,54 & 9,21 & 76,31 & $8,67 \mathrm{~A}$ \\
\hline Sorgo & 24,46 & 66,43 & 9,11 & 76,96 & $4,49 B$ \\
\hline \multicolumn{6}{|l|}{ Tipo de feno } \\
\hline $\mathrm{FFL}$ & 24,62 & 66,14 & 9,24 & 76,91 & $7,75 \mathrm{~A}$ \\
\hline $\mathrm{FC}$ & 24,09 & 66,83 & 9,08 & 76,36 & $5,41 \mathrm{~B}$ \\
\hline \multicolumn{6}{|l|}{ Efeitos - ANOVA, p -valor } \\
\hline Tratamento & 0,6353 & 0,7129 & 0,2258 & 0,8549 & $<0,0001$ \\
\hline Fonte de energia (FE) & 0,7192 & 0,8581 & 0,4893 & 0,7606 & $<0,0001$ \\
\hline Tipo de feno (TF) & 0,3707 & 0,2849 & 0,2422 & 0,7933 & $<0,0001$ \\
\hline FE $\times$ TF & 0,3112 & 0,4037 & 0,6348 & 0,3239 & 0,1092 \\
\hline
\end{tabular}

$C V=$ Coeficiente de variação. *Diferente em relação ao controle pelo teste de Dunnet $(p<0,05)$. 
no ovo varia em função de diversos fatores, sendo um dos mais importantes, a alimentação. Dada a diferente composição química de cada componente do ovo, a deficiência de determinado nutriente na ração pode, dentro de certos limites, causar redução no percentual de um dos constituintes com o conseqüente aumento de outro. Assim, enquanto os níveis de aminoácidos e de proteínas influenciam o componente clara, o teor de ácido linoléico altera a proporção de gema. Por sua vez, a formação da casca é afetada pela ingestão e absorção de minerais, principalmente de Ca e P.

Nesse contexto, pode-se deduzir que o ajuste na quantidade de ração consumida pelas aves foi suficiente para manter a ingestão adequada dos nutrientes essenciais à formação de cada um dos constituintes do ovo.

Na avaliação da coloração das gemas, observou-se que a ração controle promoveu pigmentação das gemas inferior em relação á obtida com ração à base de milho e inclusão de FFL e superior a dos demais tratamentos.

As rações à base de sorgo, independente do feno utilizado, proporcionaram gemas de pigmentação inferior às obtidas com as rações á base de milho. Por sua vez, a inclusão de FFL, na ração contendo milho ou sorgo, resultou em maior pigmentação das gemas em relação à inclusão de FC.

Os resultados obtidos indicam que o FFL tem maior potencial de pigmentação das gemas do que o FC. Provavelmente, a maior proporção de pigmentos carotenóides do FFL deve ter contribuído para isso.

Segundo Moreira (2008), o FFL apresenta maior teor de EE (5,70\%) em relação ao $\mathrm{FC}(3,59 \%)$ devido à maior concentração de pigmentos carotenóides nesse primeiro ingrediente que, por sua vez, está associado a mais alta proporção de folha empregada no preparo do mesmo. Na obtenção do FFL

\section{BIBLIOGRAFIA}

Albino, L.F.T.; Nery, L.R.; Vargas Júnior, J.G. e Silva, J.H.V. 2005. Criação de frango e galinha são utilizadas somente as folhas, parte do vegetal mais rica em carotenos, enquanto na confecção do FC se emprega toda a planta - folhas e talos tenros.

$\mathrm{Na}$ literatura são encontrados relatos sobre as características dos ovos de aves alimentadas com rações contendo fenos que se assemelham, em alguns aspectos, aos observados na presente pesquisa. Normalmente, ocorre intensificação na cor da gema em função da alta concentração de carotenóides nas folhas, porém, não se verifica influência dos níveis de inclusão dos fenos utilizados nas demais características dos ovos. Odunsi et al. (2002) relataram alterações, apenas, no índice e na cor da gema, com o incremento do nível de inclusão do feno de Gliricidia sepium na ração. Odunsi (2003) verificou que, dos parâmetros estudados, somente a gema apresentou-se mais amarela à medida que os níveis de feno de Lablab purpureus na ração cresceram.

Como o nível de pigmentação das gemas depende diretamente da alimentação recebida, ficou demonstrado que o uso de FFL nas rações de poedeiras apresenta maior viabilidade que o FC, para esse fim. A informação sobre a superioridade do FFL como fator pigmentante, deve ser bem aproveitada pelos produtores de ovos, principalmente, por aqueles que exploram aves em sistema caipira, visto que os consumidores desse tipo de produto exigem uma coloração alaranjada intensa da gema (Albino et al., 2005).

\section{CONCLUSÃO}

Independente do cereal utilizado como fonte de energia, a inclusão de $2 \%$ de feno de folha de leucena (FFL) ou de cunhã (FC) resulta em desempenho semelhante, porém, o FFL apresenta maior capacidade de pigmentação das gemas.

Caipira. Avicultura alternativa. $2^{\mathrm{a}}$. ed. Aprenda Fácil Editora. Viçosa, MG. 208 pp. 
Alcântara, P.B. e Bufarah, G. 1988. Plantas forrageiras: gramíneas e leguminosas. $5^{\mathrm{a}}$. ed. Nobel. São Paulo. 162 pp.

Araújo Filho, J.A.; Gadelha, J.A.; Pereira, R.M.A. e Sousa, P.Z. 1981. Flutuações de alguns parâmetros quantitativos e qualitativo da Clitoria ternatea. Reunião Anual da Sociedade Brasileira de Zootecnia, 18. Anais.... SBZ. Goiânia. pp. 73.

Arruda, A.M.V. de; Melo, A.S.; Oliveira, V.R.M. de; Souza, D.H.; Dantas, F.D.T. e Oliveira, J.F. de. 2010. Avaliação nutricional do feno de leucena com aves caipiras. Acta Vet Brasilica, 4: 162167.

Braga, C.V.P.; Fuentes, M.F.F.; Freitas, E.R.; Carvalho, L.E. de; Sousa, F.M. de e Bastos, S.C. 2005. Efeito da inclusão do farelo de coco em rações para poedeiras comerciais. Rev Bras Zootecn, 34: 76-80.

Brum Júnior, B.S.; Lemos, I.T.P. de; Zanella, I.; Rosa, A.P.; Carvalho, E.H.; Batista, I.M. e Magon, L. 2007. Utilização do farelo de arroz integral na dieta para poedeiras UFSM-V 2003 na fase de produção. Rev Bras Agrociênc, 13: 541-546.

D'Mello, J.P.F.; Acamovic, T. and Walker, A.G. 1987. Evaluation of leucaena leaf meal for broiler growth and pigmentation. Trop Agric (Trinidad), 64: 33-35.

Esonu, B.O.; Emenalom, O.O.; Udedibie, A.B.I.; Anyanwu, A.; Madu, U. and Inyang, A. O. 2005. Evaluation of neem (Azadirachta indica) leaf meal on performance, carcass characteristics and egg quality of laying hens. Int J Agric Rural Dev, 6: 208-212.

Esonu, B.O.; Azubuike, J.C. and Ukwu, H.O. 2004. Evaluation of Microdesmis puberula leaf meal as feed ingredient in laying hen diets. Int $\mathrm{J}$ Poult Sci, 3: 96-99.

Garcia, A.F.Q.M.; Murakami, A.E.; Furlan, A.C.; Massuda, E.M.; Potença, A. e Rojas, I.C.O. 2011. Milheto na alimentação de poedeiras. Acta Sci Anim Sci, 33: 73-75.

Grobas, S. y Mateos, G.G. 1996. Influencia de la nutricion sobre la composición nutricional del huevo. In: Curso de especialización FEDNA, 12. FEDNA. Madrid. pp. 219-244.

Janssen, W.M.M.A. and Carré, B. 1989. Influence of fiber on digestibility of poultry feeds. In: Cole, D.J.A.; Haresign. W. (Eds.). Recent developments in poultry nutrition. Butterworths. London. pp. 78-93.

Leeson, S. and Summers, J.D. 2001. Nutrition of the chicken. $4^{\text {th }}$ ed. University Books. Canada. $591 \mathrm{pp}$.

Moreira, R.F. 2008. Avaliação nutricional de fenos utilizados na alimentação de poedeiras. Dissertação (Mestrado em Zootecnia). Universidade Federal do Ceará. Ceará. 46 pp.

Moreno, J.O.; Espíndola, G.B.; Santos, M.S.V.; Freitas, E.R.; Gadelha, A.C. e Silva, F.M.C. 2007. Desempenho e qualidade dos ovos de poedeiras comerciais, alimentadas com dietas contendo sorgo e páprica em substituição ao milho. Acta Sci Anim Sci, 29: 159-163.

Odunsi, A.A. 2003. Assessment of lablab (Lablab purpereus) leaf meal as a feed ingredient and yolk colouring agent in the diet of layers. Int J Poult Sci, 2: 71-74.

Odunsi, A.A.; Ogunleke, M.O.; Alagbe, O.S. and Ajani, T.O. 2002. Effect of feeding Gliricidia sepium leaf meal on the performance and egg quality of layers. Int J Poult Sci, 1: 26-28.

Oliveira, P.B.; Murakami, A.E. e Garcia, E.R.M. 2000. Influência de fatores antinutricionais da leucena (Leucaena leucocephala) e do feijão guandu (Cajanus cajan) sobre epitélio intestinal e desempenho de frangos de corte. Rev Bras Zootecn, 6: 1759-1769.

Rostagno, H.; Albino, L.F.T.; Donzele, J.L.; Gomes, P.C.; Oliveira, R.F. de; Lopes, D.C.; Ferreira, A.S. e Barreto, S.L.T. 2005. Tabelas brasileiras para aves e suínos. Composição de alimentos e exigências nutricionais. $2^{\mathrm{a}}$. ed. UFV/DZO. Viçosa. 186 pp.

Sakomura, N.K. e Rostagno, H.S. 2007. Métodos de pesquisa em nutrição de monogástricos. FUNEP. Jaboticabal, SP. 283 pp.

SAS Institute. 2000. SAS software: user's guide. Version 8. 2. ed. SAS Institute Inc. Cary. USA.

Silva, J.H.V.; Albino, L.F.T. e Godói, M.J.S. 2000. Efeito do extrato de urucum na pigmentação da gema dos ovos. Rev Bras Zootecn, 29: 14351439.

Archivos de zootecnia vol. 63, núm. 241, p. 190. 